\title{
ANN MODEL OF STRESS-STRAIN RELATIONSHIP FOR ALUMINIUM SPONGE IN UNIAXIAL COMPRESSION
}

MAREK DUDZIK

Cracow University of Technology, Faculty of Electrical and Computer Engineering, Cracow, Poland

e-mail: marekdudzik@pk.edu.pl

Anna Mazgorzata StręK

Cracow University of Technology, Faculty of Civil Engineering, Cracow, Poland

e-mail: anna.strek@pk.edu.pl

In this article, we present a proposition of a model of the compressive behaviour of open-cell aluminium with relation to the material apparent density. The research was based on experimental data from uniaxial compression tests conducted for two sample lots. These results were analysed with the use of neural networks in a specially designed algorithm. The main criterion for choosing a satisfactory approximation was mean absolute relative error MARE $<5 \%$. As a result of the analysis, the sought relation was extracted and is presented as a proposition of a new ANN model of the compressive stress-strain relationship for aluminium sponge.

Keywords: artificial neural network design, compressive behaviour, open-cell aluminium, model equation

\section{Introduction}

Cellular and porous metals are a well-known group of smart materials which play role in a wide variety of applications from medicine, through civil engineering, electronics, automotive and space industry to even battle fields, e.g. (García-Moreno, 2016; Kränzlin and Niederberger, 2015). Specific applications are of course dependent on individual properties which are a consequence of the skeletal material and special porous morphology. In turn, the individual characteristic porous structure is a derivative of the production method. Now, against this quite broadly outlined background, it is time to introduce in more detail the material chosen for the presented study and the authors' approach to contribute to scientific discussion regarding it. The article reports a part of a wider research of an open-cell metal sponge with an aluminium skeleton. The material was self-produced by investment casting. The overall study comprises various aspects, but here we focus on compressive behaviour and its description.

Usually, the starting point in a discussion of compressive behaviour of an open-cellular metal is the following general formula (Gibson and Ashby, 1988) which binds some characteristic, interesting for a designer, parameter with the sponge relative density $\rho / \rho_{s}$

$$
\frac{\text { cellular material property }}{\text { skeleton property }}=C\left(\frac{\rho}{\rho_{s}}\right)^{n}
$$

where $C$ and $n$ are to be determined experimentally. However, only few values, e.g. plastic collapse strength $\sigma_{p l . c}$ or boundary between plastic collapse and densification $\sigma^{*}$, can be predicted based on the formulae from (Gibson and Ashby, 1988). The usage of the referred approach might be limited due to the necessity of knowledge of experimentally derived constants, as well as the 
knowledge of skeleton characteristics such as the yield point, modulus, density, which are also included in the formulae.

In this study, the authors employ a modern computational method, namely artificial intelligence, for the purpose of finding a mathematical formula for description of compressive behaviour of the open-cell aluminium. The resulting approximation is supposed to free the designer from the necessity of calibration of experimental constants, as well as the knowledge of skeleton characteristics, and provide the possibility of finding the whole range of stresses instead just single values. The idea of making use of artificial intelligence was first encouraged by the fact that neural networks were reported useful in mechanics of materials (e.g., Potrzeszcz-Sut and Pabisek, 2014; Sumelka and Łodygowski, 2013). The possibility of using neural networks specifically for the studied problem was preliminary positively verified by the authors in (Stręk et al., 2019a).

The overall form of the sought relation was chosen so as to bind stress with strain and apparent density

$$
\sigma=f(\varepsilon, \rho)
$$

\section{Material and uniaxial compression experiments}

This Section will be limited only to necessary information, all details can be found in separate publications (Stręk et al., 2019b; Kasza et al., 2016). The studied material was an open-cellular aluminium sponge, two sample lots were investigated: the prototype lot $P$ (with minor structural imperfections like swellings of struts, small number of semi-closed cells, mould residuals sunk inside) and the regular lot $R$ without visible structural flaws. The material had PPI=5.4-6.2 in both groups. Apparent density varied over the specimens: $\rho=0.32-0.36 \mathrm{~g} / \mathrm{cm}^{3}$ in $P$ and $\rho=0.36-0.56 \mathrm{~g} / \mathrm{cm}^{3}$ in $R$. Compression experiments were uniaxial, quasistatic and were conducted at room temperature using Zwick $145520 \mathrm{kN}$ machine with programme TestExpert II. The obtained results are depicted together with the modelling in Fig. 2.

\section{The neural network used for modelling}

\subsection{Data for the network}

The results from compression experiments as well as specimens apparent densities and the auxiliary parameter-indicator of the lot were used as data for training the neural network. For each sample, 1000 experimental values of strain (expressed as a decimal number, not \%) and respective stress (expressed in $\mathrm{MPa}$ ) were taken. Stresses and strains were defined according to (ISO 13314:2011):

$\sigma$ - stress which is the compressive force divided by the initial cross-section area perpendicular to the loading direction,

$\varepsilon$ - strain which is the overall compressive displacement divided by the initial height of the test specimen.

The additional parameters - lot indicators $l_{P}$ and $l_{R}$-assumed value 1 if the sample belonged to the given group and 0 in the opposite case. Arguments entering the neural network were arranged into vectors $\mathbf{A}_{i}=\left[\varepsilon_{i}, \rho_{i}, l_{P . i}, l_{R . i}\right]^{\mathrm{T}}$, and the corresponding stress from the $i$-th experimental data set was assumed as the respective target $t_{i}=\sigma_{i}$. The specific relation sought by the network had then the following general form (augmented with respect to assumed general relation (1.2))

$$
\sigma=f\left(\left[\varepsilon, \rho, l_{P}, l_{R}\right]^{\mathrm{T}}\right)
$$


The data was normalised. Calculations were performed in the Matlab environment, so the inbuilt function "mapminmax" was utilised for this purpose (Demuth et al., 2009). It is equivalent to

$$
V^{\prime}=\frac{V-V_{\min }}{V_{\max }-V_{\min }}\left(V_{\max }^{\prime}-V_{\min }^{\prime}\right)+V_{\min }^{\prime}
$$

where: $V^{\prime}$ is the new value, $V$ - original value, $V_{\max }, V_{\min }$ - original interval limits, $V_{\max }^{\prime}, V_{\min }^{\prime}$ - new limits, here: -1 and 1 . The vectors $\mathbf{A}_{i}$ were normalised before entering the network, and the network outputs were post-processed by a reverse relation after computations.

\subsection{The network architecture and learning parameters}

The neural network structure type and transfer functions were chosen in accordance with the recommendations for a nonlinear function approximation from the literature (Hagan et al., 2014). Also, a preliminary work of the authors (Stręk et al., 2019a) confirmed this choice and additionally helped in the assumption of learning parameters. The number of neurons in the hidden layer was determined in a specially devoted research (Dudzik, 2019) to fulfil the expected accuracy.

The artificial neural network was assumed as a feedforeward network with one hidden layer with 7 neurons and one output layer with 1 neuron. In the hidden layer, the activation function was hyperbolic tangent sigmoid-tansig. In the output layer, the transfer function was linear-purelin. The learning process included three stages: training, validation and test. Experimental data was uniformly distributed between the stages in the respective proportions: $60 \%, 20 \%$ and 20\%. The training algorithm was Levenberg-Marquardt (Madsen et al., 2004) with the mean square error as a performance function with its goal set as 0 . The minimum performance gradient was $10^{-10}$, maximum validation failures were set as 12 . The learning rate was assumed as 0.01 , momentum as 0.9 and the maximum number of epochs to train -100000 .

\subsection{Performance of the network}

The presented network approximated the experimental results with a good engineering accuracy - the mean absolute relative error $M A R E=4.8 \%$. Figure 1 shows the outputs from the network plotted against targets, i.e. approximate stresses vs. experimental stresses. A linear regression between them was performed - the obtained fits are depicted left to each of the graphs and the Pearson coefficients are above them. Figure 2 depicts the experimental data and the network approximation of stresses with regard to strain and density. Additionally, the values of errors are also visible in light blue.

(a)

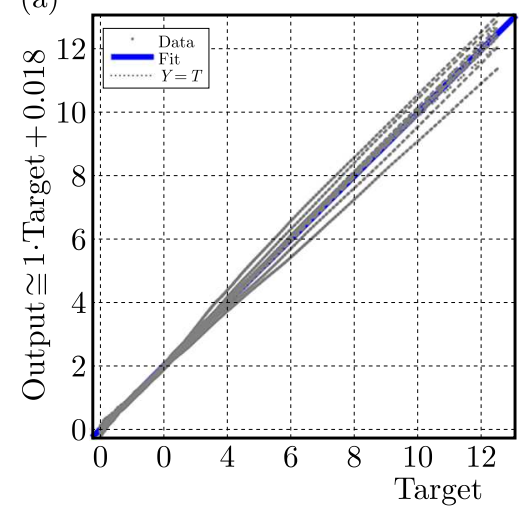

(b)

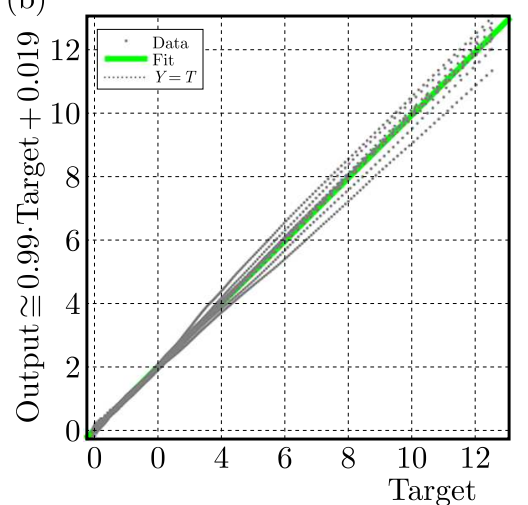

(c)

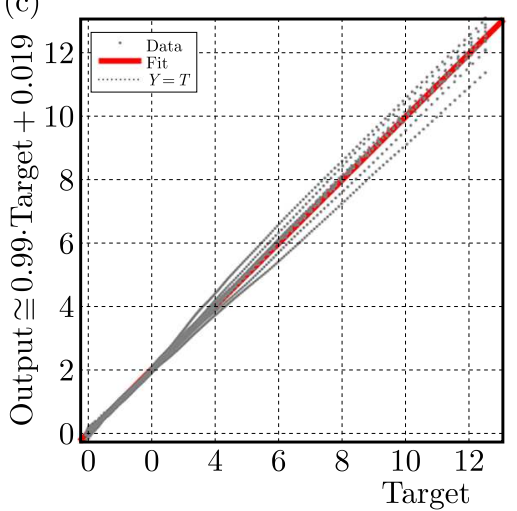

Fig. 1. A linear fit between network outputs and targets for all learning stages separately 


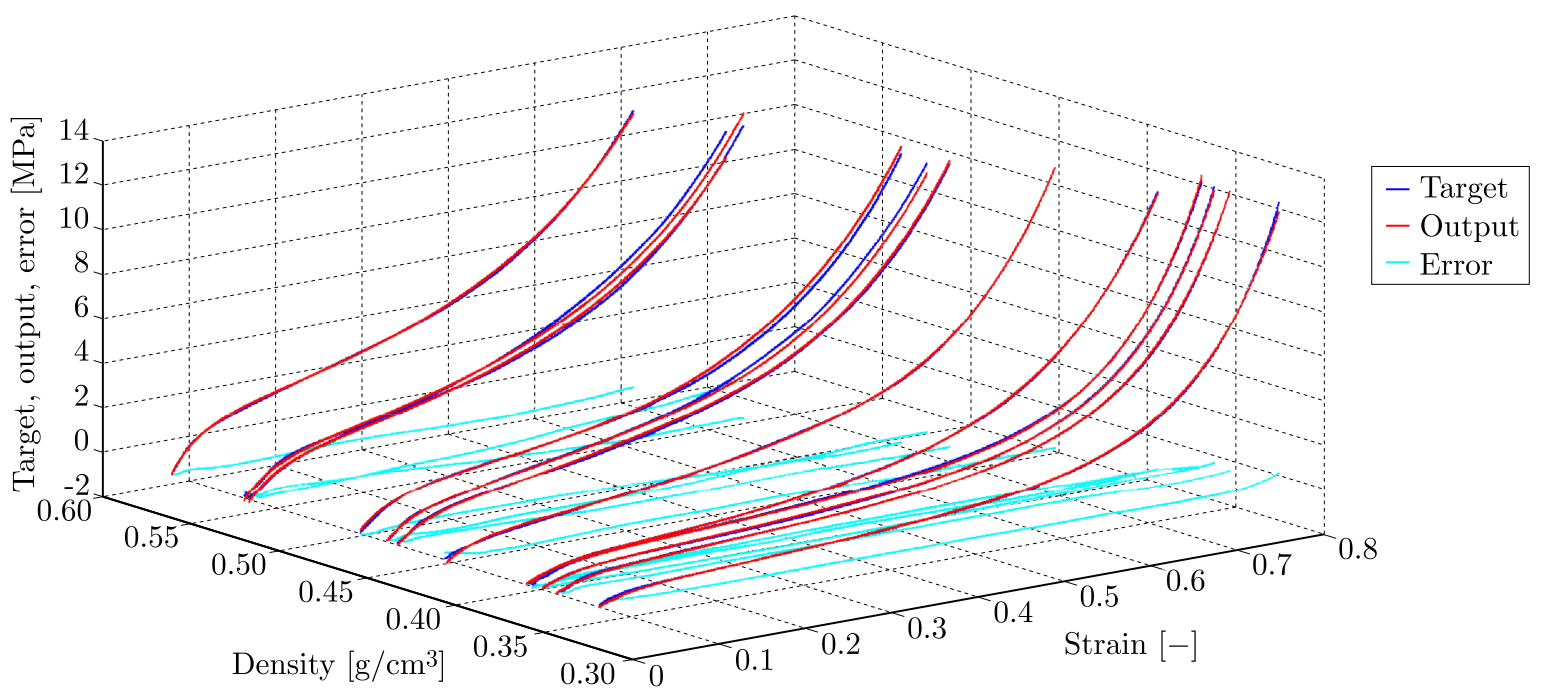

Fig. 2. Experimental and approximated data with respect to strain and apparent density

\section{Results - equation for the stress-strain relation}

The following general relation approximating assumed Eq. (3.1) was obtained

$$
\sigma=\operatorname{mapminmax}^{-1}\left\{f_{\text {activ }}^{\{1\}}\left[\mathbf{W}^{\{1\}} \cdot\left(\mathbf{f}_{\text {activ }}^{\{2\}}(\mathbf{a})\right)+B^{\{1\}}\right]\right\}
$$

According to the assumptions and results of the present research, the first activation function $f_{\text {activ }}^{\{1\}}$ and bias $B^{\{1\}}$ are in this case equal to

$$
B^{\{1\}}=72.060 \quad f_{\text {activ }}^{\{1\}}(x)=1 \cdot x
$$

Now, Eq. (4.1) can be expressed in the following, ready-for-use form

$$
\sigma=\operatorname{mapminmax}^{-1}\left(\mathbf{W}^{\{1\}} \cdot\left(\mathbf{f}_{\text {activ }}^{\{2\}}(\mathbf{a})\right)+72.060\right)
$$

where

$$
\begin{aligned}
& \mathbf{W}^{\{1\}}=[0.584,73.278,-0.701,-72.587,0.085,138.775,-60.245] \\
& \mathbf{f}_{\text {activ }}^{\{2\}}(\mathbf{a})=\frac{\mathrm{e}^{2 \mathbf{a}}-1}{\mathrm{e}^{2 \mathbf{a}}+1} \\
& \mathbf{a}=\mathbf{a}\left(\left[\varepsilon, \rho, l_{P}, l_{R}\right]^{\mathrm{T}}\right)=\mathbf{W}^{\{2\}} \cdot \operatorname{mapminmax}\left(\left[\varepsilon, \rho, l_{P}, l_{R}\right]^{\mathrm{T}}\right)+\mathbf{B}^{\{2\}} \\
& \mathbf{W}^{\{2\}}=\left[\begin{array}{cccc}
-1.021 & 10.703 & -1.536 & 2.903 \\
5.711 & -0.241 & 0.037 & 0.129 \\
0.789 & 7.389 & 2.178 & -2.087 \\
-0.013 & -0.001 & -1.395 & -0.012 \\
-2.669 & -5.019 & -0.141 & -0.284 \\
1.401 & 0.247 & 0.792 & 1.242 \\
1.392 & 0.288 & 0.559 & -2.962
\end{array}\right] \quad \mathbf{B}^{\{2\}}=\left[\begin{array}{c}
8.129 \\
9.098 \\
0.259 \\
0.446 \\
-0.556 \\
-3.047 \\
1.353
\end{array}\right]
\end{aligned}
$$

Also, two functions should be understood as follows:

- mapminmax - Matlab inbuilt normalisation function "mapminmax", equivalent to Eq. $(3.2)$

- $\operatorname{mapminmax}^{-1}$ - Matlab inbuilt denormalisation, equivalent to the reverse of Eq. (3.2). 
The above formula, Eqs. (4.1) and (4.2), can be used to generate data in the domain ranges defined in Table 1. The density interval $\rho=0.36-0.405$ is a transition between the ranges of samples $P$ and $R$ and, due to this fact, it is problematic for the model. Also, it should be noted that the largest values of absolute relative errors from the network were obtained for the beginnings of the approximated experimental curves (up to $\varepsilon \approx 0.025$ for $R$ and $\varepsilon \approx 0.0375$ for $P$ ). This should be kept in mind when using the here proposed model, and the initial range of strains should be preferably modelled in an augmented way. A proposition of such an improvement is currently elaborated by the authors (Dudzik and Stręk, 2020).

Table 1. Domain ranges for the equation modelling the compression of an open-cell aluminium

\begin{tabular}{|c|c|c|}
\hline Apparent density $\rho\left[\mathrm{g} / \mathrm{cm}^{3}\right]$ & Strain $\varepsilon$ & Lot indicators \\
\hline \hline $0.32-0.36$ & up to 0.8 & $l_{P}=0$ and $l_{R}=1$ \\
\hline $0.405-0.515$ & up to 0.65 & $l_{P}=1$ and $l_{R}=0$ \\
\hline $0.52-0.56$ & up to 0.58 & $l_{P}=1$ and $l_{R}=0$ \\
\hline
\end{tabular}

\section{Conclusions}

Thanks to the approach using neural networks, the authors obtained an analytical function which allows one to determine single curves for chosen densities or a surface for a range of densities to model the compressive behaviour of an open-cell aluminium with PPI $\approx 5.8$. The model was generated based on experimental data for prototype and regular samples. For these data, the mean absolute relative error assured good engineering precision $<5 \%$. Further development of the research is going to comprise the improvement of approximation for the initial strain interval. Also, investigation of materials with other structures or skeletal materials would be valuable.

\section{Acknowledgements}

The authors would like to acknowledge that computational modelling and partially experimental tests were supported by Cracow University of Technology. Also, production of aluminium samples and experimental tests were in part supported by the AGH University of Science and Technology (grant No. 15.11.130.488). Recticel Flexible Foams, Belgium, provided precursors for the production of aluminium samples.

\section{References}

1. Demuth H., Beale M., Hagan M., 2009, Neural Network Toolbox 6 User's Guide, MathWorks Inc.

2. Dudzik M., StręK A.M., 2020, ANN architecture specifications for modelling of open-cell aluminium under compression, Mathematical Problems in Engineering, in press

3. García-Moreno F., 2016, Commercial applications of metal foams: their properties and production, Materials, 9, 85, DOI:10.3390/ma9020085

4. Gibson L.J., Ashby M.F., 1988, Cellular Solids. Structure and Properties, Pergamon Press

5. Hagan M.T., Demuth H.B., Beale M.H., De Jesus O., 2014, Neural Network Design, eBook

6. ISO 13314:2011 Mechanical testing of metals - Ductility testing - Compression test for porous and cellular metals

7. Kasza P., Lipowska B., Pęcherski R.B., Stręk A.M., Wańczyk K., 2016, Compression of open-cell aluminium, Engineering Transactions, 64, 4, 629-634 
8. Kränzlin N., Niederberger M., 2015, Controlled fabrication of porous metals from the nanometer to the macroscopic scale, Materials Horizons, 2, 4, 359-377, DOI: 10.1039/C4MH00244J

9. Madsen K., Nielsen H.B., Tingleff O., 2004, Methods for non-linear least squares problems, [In:] Informatics and Mathematical Modelling Technical University of Denmark, available online: http://www2.imm.dtu.dk/pubdb/views/edoc_download.php/3215/pdf/imm3215.pdf (accessed on 15th Oct 2018)

10. Potrzeszcz-Sut B., PABISEK E., 2014, ANN constitutive material model in the shakedown analysis of an aluminium structure, CAMES, 21, 49-58

11. StręK A.M., Dudzik M., Kwiecień A., Wańczyk K., Lipowska B., 2019a, Verification of application of ANN modelling for compressive behaviour of metal sponges, Engineering Transactions, 67, 271-288

12. StręK A.M., Lipowska B., WAŃCZYK K., 2019b, Selected aspects of manufacturing of aluminium sponge, Archives of Metallurgy and Materials, 3, 1145-1150

13. Sumelka W., Łodygowski T., 2013, Reduction of the number of material parameters by ANN approximation, Computational Mechanics, 52, 2, 287-300

Manuscript received November 4, 2019; accepted for print January 16, 2020 